\title{
论 文
}

\section{不同晶型 $\mathrm{MnO}_{2}$ 纳米线的催化苂光传感研究}

\author{
吴振龙, 鸟佳佳, 孙向英, 刘斌, 沈江珊* \\ 华侨大学材料科学与工程学院, 厦门 361021 \\ *通讯作者, E-mail: jsshen@hqu.edu.cn
}

收稿日期: 2017-04-16; 接受日期: 2017-07-31; 网络版发表日期: 2017-09-15

国家自然科学基金(编号: 21275059, 21377124, 21575044)、福建省自然科学基金(编号: 2015J01054, 2016J01062, 2017J01015)、华侨大学中 青年教师科技创新资助计划项目(编号: ZQN-YX303)以及华侨大学人才引进科研启动项目(编号: 15BS202)

\begin{abstract}
摘要采用水热合成法, 制备了 4 种不同晶型 $(\alpha 、 \beta 、 \gamma 、 \delta)$ 的 $\mathrm{MnO}_{2}$ 纳米线. 以邻苯二胺 $(\mathrm{OPD})$ 为底物分子, 系统地研究了不同晶型 $\mathrm{MnO}_{2}$ 纳米线的催化生荧反应. 采用透射电子显微镜(TEM)、扫描电子显微镜( SEM)、 X射线粉末衍射 (XRD)、光电子能谱(XPS)、核磁共振、质谱和电化学方法等实验手段进行表征研究. 实验 结果表明, $\beta-\mathrm{MnO}_{2}$ 纳米线对 $\mathrm{OPD}$ 的催化生荧反应为最佳. 在此研究的基础上,借助甲醛的- $\mathrm{CHO}$ 基团和 $\mathrm{OPD}$ 的- $\mathrm{NH}_{2}$ 基团可发生缩合反应形成Schiff碱的性质, 进一步构建了一种新型、高选择性、灵敏的催化荧光传感 体系, 并用于食品中甲醛的检测研究.
\end{abstract}

关键词 $\mathrm{MnO}_{2}$ 纳米线, 催化生荧反应, 荧光传感, 甲醛检测

\section{1 引言}

催化反应不仅是大多数化工生产的核心, 而且是 生命体系的基础, 在众多领域中发挥着极其重要的作 用 ${ }^{[1]}$. 天然酶具有极高的底物专一性和催化效率, 是 维持生命体系运转的重要物质, 在很多研究领域如 化学/生物传感和环境化学等方面均有应用. 然而, 天 然酶存在严重不足之处. 例如, (1) 多数天然酶极易变 性, 使用条件苛刻, 不便于存储、运输和操作使用; (2) 制备和提纯天然酶的实验操作繁琐、复杂, 使其制备 成本高昂 ${ }^{[2,3]}$. 因此, 寻找高效、稳定性好、易制备和 低成本的人工模拟酶来取代天然酶, 以克服天然酶自 身存在的这些缺陷, 是拓展酶应用研究的一种有效途 径.

在众多的人工模拟酶中, 纳米模拟酶尤其引人注
目. 2007 年, 阎锡蕴课题组 ${ }^{[4]}$ 在Nature Chemistry上发 表的研究出乎人意料之外: 原本被认为是化学惰性 的 $\mathrm{Fe}_{3} \mathrm{O}_{4}$ 磁性纳米颗粒具有类似过氧化物酶的催化活 性. 此后, 研究发现, 一系列纳米材料也具有类似的 催化性质, 包括 $\mathrm{Au} 、 \mathrm{Ag} 、 \mathrm{Cu} 、 \mathrm{CuO} 、 \mathrm{CeO}_{2} 、 \mathrm{Fe}_{2} \mathrm{O}_{3}$ 、 $\mathrm{FeS} 、 \mathrm{CuS}$ 、碳纳米管、纳米碳点以及氧化石墨烯 等纳米材料 ${ }^{[5 \sim 15]}$. 这些纳米模拟酶也被称为“纳米酶 (Nanoenzyme)”. 借助催化生荧反应或催化生色反应, 通过研究这些纳米酶, 有力地推动了其在生物分子/ 环境污染物的检测、肿瘤细胞成像、抗菌甚至污水 处理等方面的应用. 这些纳米材料的催化活性与其本 身的纳米效应如尺寸效应和表面效应等有着密切的 关系 ${ }^{[16]}$. 因此, 这些纳米效应为优化纳米催化剂的设 计提供了可操作的途径, 即通过控制这些效应可调控 纳米材料的催化活性和底物分子的选择性. 需要指出

引用格式: 吴振龙, 邬佳佳, 孙向英, 刘斌, 沈江珊. 不同晶型 $\mathrm{MnO}_{2}$ 纳米线的催化苂光传感研究. 中国科学: 化学, 2017, 47: 1226-1232

Wu Z, Wu J, Sun X, Liu B, Shen J. Fluorescence sensing of formaldehyde based on the catalytic reaction of $\mathrm{MnO}_{2}$ nanowires modulated by different crystal structures. Sci Sin Chim, 2017, 47: 1226-1232, doi: 10.1360/N032017-00072 
的是, 目前在这些纳米材料中, 绝大多数存在着结构 复杂、多晶面混合、尺寸不均一等问题, 导致纳米材 料的表面态有着极大的差异, 从而对催化反应的结果 产生混乱、不清晰的影响, 甚至使催化活性大大降低. 这也是传统的纳米催化光化学传感研究过程中遇到 的一个瓶颈问题. 事实上, 较多研究表明, 高比例暴露 晶面是纳米材料催化活性的重要决定因素之一. 因此, 通过制备结构可控、暴露晶面明确的纳米材料, 有望 获得高催化活性的纳米催化剂, 为建立新型、灵敏、 高选择性的催化传感体系提供有效途径. 但是, 一直 以来关于这方面的研究报道非常少. 本课题组 ${ }^{[17]}$ 在之 前的研究中发现, $\beta-\mathrm{HgS}$ 纳米晶具有优秀的催化生苂 反应活性, 而 $\alpha-\mathrm{HgS}$ 纳米晶却不具有.

另一方面, 氧化锰尤其 $\mathrm{MnO}_{2}$ 是一类被广泛研 究的金属氧化物材料, 其在催化 ${ }^{[18-20]}$ 、电化学 ${ }^{[21]}$ 和 传感 ${ }^{[22,23]}$ 等领域引人注目. 但是, 就催化荧光传感方 面而言, 从晶型的角度来探究 $\alpha-、 \beta-、 \gamma-、 \delta-\mathrm{MnO}_{2}$ 的催化生荧活性, 目前鲜有报道. 本文通过水热合 成法 ${ }^{[24,25]}$, 制备了 $\alpha-、 \beta-、 \gamma-、 \delta-\mathrm{MnO}_{2}$ 4种不同晶型的 纳米线, 并以邻苯二胺 (OPD)为底物分子, 研究了 4 种 不同晶型的 $\mathrm{MnO}_{2}$ 纳米线的催化生荧反应. 基于甲醛 的-CHO基团和 $\mathrm{OPD}$ 的 $-\mathrm{NH}_{2}$ 基团可发生缩合反应形成 Schiff碱的性质, 结合 $\beta-\mathrm{MnO}_{2}$ 纳米线的催化生荧反应, 进一步构建了一种新型、灵敏、高选择性的催化苂 光传感体系, 用于食品中甲酫的检测研究.

\section{2 实验部分}

\section{1 试剂与仪器}

OPD购自上海麦克林试剂有限公司(中国); 苂光光 谱测定采用F-7000荧光光谱测仪(Hitachi公司, 日本); UV-Vis吸收光谱采用UV-2600PC光谱仪 (Shimadzu公 司, 日本). 详细的试剂与仪器可参见支持信息(网络 版)中的“实验部分”.

\section{2 实验过程}

$\alpha-\mathrm{MnO}_{2}$ 纳米线的合成操作如下: 用 $80 \mathrm{~mL}$ 超纯水 溶解0.525 $\mathrm{g} \mathrm{MnSO}_{4} \cdot \mathrm{H}_{2} \mathrm{O}$ 和 $1.25 \mathrm{~g} \mathrm{KMnO}_{4}$ 固体, 磁力摚 拌均匀后, 将溶液转移至 $100 \mathrm{~mL}$ 不锈钢反应釜中, 并 在 $160^{\circ} \mathrm{C}$ 下反应 $12 \mathrm{~h}$. 获得的产物用超纯水洗涤 5 次, 最 后在 $80^{\circ} \mathrm{C}$ 的烘箱下干燥。除了前驱体和反应温度、 反应时间不同以外, 其他几种晶型 $\mathrm{MnO}_{2}$ 纳米线的制备
方法均与 $\alpha-\mathrm{MnO}_{2}$ 纳米线相似.

$\beta-\mathrm{MnO}_{2}$ 纳米线是将 $1.69 \mathrm{~g} \mathrm{MnSO}_{4} \cdot \mathrm{H}_{2} \mathrm{O}$ 和 $2.28 \mathrm{~g}$ $\left(\mathrm{NH}_{4}\right)_{2} \mathrm{~S}_{2} \mathrm{O}_{8}$ 在 $140^{\circ} \mathrm{C}$ 下反应 $12 \mathrm{~h}$ 所得; $\gamma-\mathrm{MnO}_{2}$ 纳米线是 将3.375 $\mathrm{g} \mathrm{MnSO}_{4} \cdot \mathrm{H}_{2} \mathrm{O}$ 和 $4.575 \mathrm{~g}\left(\mathrm{NH}_{4}\right)_{2} \mathrm{~S}_{2} \mathrm{O}_{8}$ 在 $90^{\circ} \mathrm{C}$ 下反 应 $24 \mathrm{~h}$ 所得; $\delta-\mathrm{MnO}_{2}$ 纳米线是将 $0.275 \mathrm{~g} \mathrm{MnSO}_{4} \cdot \mathrm{H}_{2} \mathrm{O}$ 和 $1.5 \mathrm{~g} \mathrm{KMnO}_{4}$ 在 $240^{\circ} \mathrm{C}$ 下反应 $24 \mathrm{~h}$ 所得.

$\mathrm{MnO}_{2}$ 纳米线的合成以及催化生苂反应等详细的 实验步骤可参见支持信息(网络版)中的“实验部分”.

\section{3 结果与讨论}

采用水热合成法 ${ }^{[24,25]}$, 并通过控制 Mn 前驱体的浓 度、氧化剂的种类和浓度以及反应温度, 制备出 4 种 不同晶型的 $\mathrm{MnO}_{2}$ 纳米线. $\mathrm{X}$ 射线粉末衍射 $(\mathrm{XRD})$ 实验 结果表明, 成功制备了 4 种晶型 $\mathrm{MnO}_{2}$, 分别为 $\alpha-, \beta-, \gamma-$, $\delta-\mathrm{MnO}_{2}$ (图1). 透射电子显微镜(TEM) 和扫描电子显微 镜 (SEM) 实验结果揭示, 所制备的 $\alpha-、 \beta-、 \gamma-、 \delta-\mathrm{MnO}_{2}$ 均具有纳米线的形貌, 尽管其直径大小不同(图 $\mathrm{S} 1$ 和 $\mathrm{S} 2$, 网络版支持信息). 光电子能谱(XPS)实验数据表 明, $\alpha-、 \beta-、 \gamma-、 \delta-\mathrm{MnO}_{2}$ 纳米线中 $\mathrm{Mn}$ 的 $2 \mathrm{P}_{1 / 2}$ 和 $2 \mathrm{P}_{3 / 2}$ 轨 道结合能(图2)都与文献中 ${ }^{[26,27]} \mathrm{MnO}_{2}$ 的 $\mathrm{Mn} 2 \mathrm{P}$ 轨道结 合能非常接近, 而且 $3 \mathrm{~S}$ 轨道结合能差均为 $4.7 \mathrm{eV}$ (图 $\mathrm{S} 3)$, 这表明所制备的 $\mathrm{MnO}_{2}$ 纳米线中的 $\mathrm{Mn}$ 均主要以 +4 价态存在 ${ }^{[28,29]}$.

以 $\mathrm{OPD}$ 为底物分子, 研究 $\mathrm{MnO}_{2}$ 纳米线的催化生 荧反应. 将一定量的 $\alpha-、 \beta-、 \gamma-、 \delta-\mathrm{MnO}_{2}$ 纳米线溶 液、 $\mathrm{pH} 7.4$ 的 $0.2 \mathrm{M}$ PB缓冲溶液 $\left(\mathrm{NaH}_{2} \mathrm{PO}_{4}-\mathrm{Na}_{2} \mathrm{HPO}_{4}\right)$ 和 $\mathrm{OPD}$ 溶液混合(保持最终 $\mathrm{Mn}$ 浓度相同), 然后将混 合溶液在 $50^{\circ} \mathrm{C}$ 下加热 $30 \mathrm{~min}$. 结果发现, 这 4 种 $\mathrm{MnO}_{2}$ 纳米线均具有催化生荧反应性质. 进一步研究其苂 光光谱, 结果表明, $\mathrm{MnO}_{2}$ 纳米线的催化生苂反应活性 按 $\beta-\mathrm{MnO}_{2}>\gamma-\mathrm{MnO}_{2}>\alpha-\mathrm{MnO}_{2}>\delta-\mathrm{MnO}_{2}$ 顺序递减(图 S4). 这一实验结果进一步支持了不同晶型纳米材料的催 化生苂反应的活性是不同的推论 ${ }^{[17,30]}$. 我们认为, 这 可能是由不同晶型 $\mathrm{MnO}_{2}$ 纳米线的主要暴露晶面不同 造成的 ${ }^{[17]}$. XRD实验结果也表明, $\beta-\mathrm{MnO}_{2}$ 纳米线由 (110) 晶面占主导, 而 $\alpha-、 \gamma-、 \delta-\mathrm{MnO}_{2}$ 纳米线的主导晶 面则不明显. 这一晶面结果也可由高分辨透射电子 显微镜(HRTEM)实验进一步证实(图3). 根据参考文 献[31,32]的方法, 计算 $\beta-\mathrm{MnO}_{2}$ 纳米线的(110)晶面所占 比例约为 $35.8 \%$, 表明其具有相对较高的晶面暴露比. 事实上, 相关的研究报道也已表明, $\beta-\mathrm{MnO}_{2}$ 纳米线的 


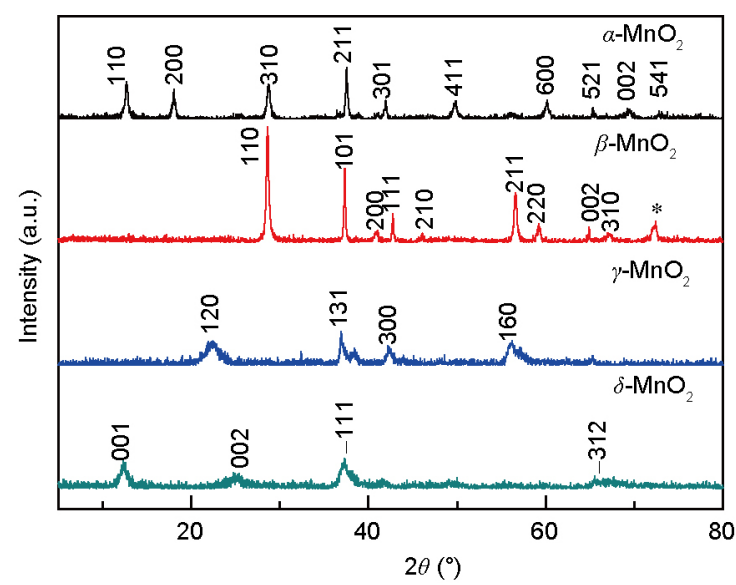

图 $1 \alpha-、 \beta-、 \gamma-、 \delta-\mathrm{MnO}_{2}$ 纳米线的XRD图(网络版彩图)

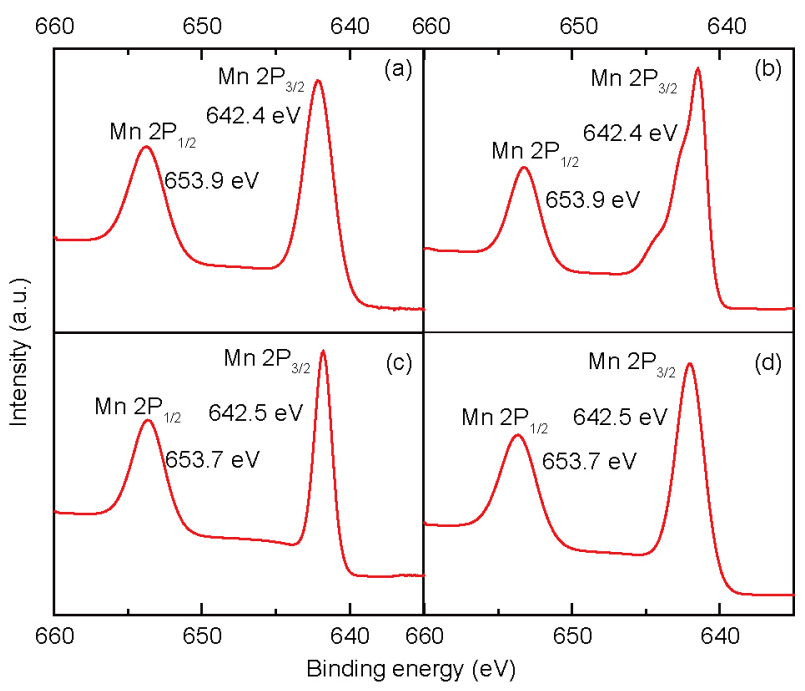

图 $2 \alpha-(\mathrm{a}) 、 \beta-(\mathrm{b}) 、 \gamma-(\mathrm{c}) 、 \delta-\mathrm{MnO}_{2}(\mathrm{~d})$ 纳米材料 $\mathrm{Mn} 2 \mathrm{P}$ 轨 道的XPS图(网络版彩图)

(110) 晶面具有较高的催化活性 ${ }^{[18-20]}$. 为了进一步探究 $\mathrm{MnO}_{2}$ 形貌对其催化生苂活性是否有影响, 也以 OPD 为底物分子, 研究 $\mathrm{MnO}_{2}$ 纳米颗粒的催化生荧反应. 结 果揭示, 相对于 $\beta-\mathrm{MnO}_{2}$ 纳米线, $\mathrm{MnO}_{2}$ 纳米颗粒的催化 生苂活性则更低一些(图S5和S6). 比表面积的研究结 果表明, $\alpha-、 \beta-、 \gamma-、 \delta-\mathrm{MnO}_{2}$ 纳米线的比表面积测定 分别为 $54.483 、 14.526 、 67.687$ 和 $151.248 \mathrm{~m}^{2} / \mathrm{g}$. 因此, $\mathrm{MnO}_{2}$ 纳米线的比表面积顺序为 $\delta->\gamma_{-}>\alpha->\beta$ - 与其催化 生荧活性的顺序 $(\beta->\gamma->\alpha->\delta$-) 没有必然联系, 从而也 说明 $\mathrm{MnO}_{2}$ 纳米线的催化生荧活性并不受其比表面积 影响, 而主要是受其主要暴露晶面的影响.

对照实验支持了 $\mathrm{MnO}_{2}$ 纳米线在生荧反应中起到
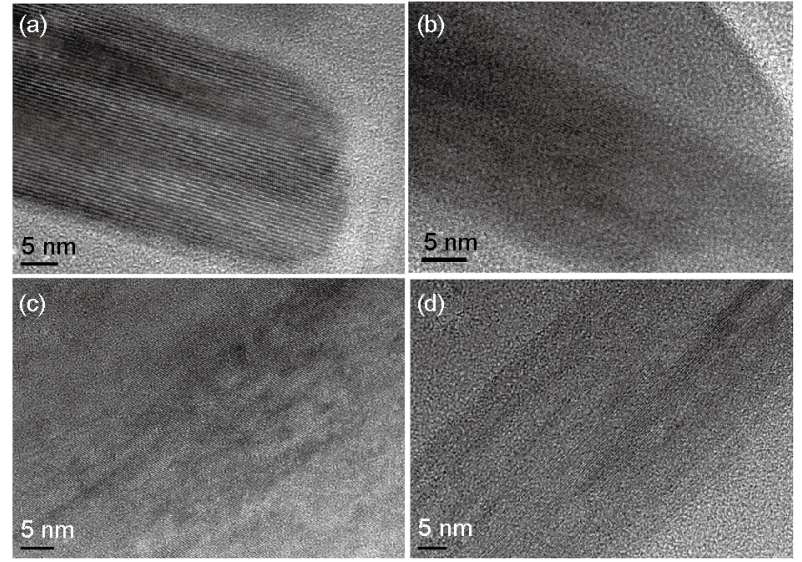

图 $3 \alpha-(\mathrm{a}) 、 \beta-(\mathrm{b}) 、 \gamma-(\mathrm{c}) 、 \delta-\mathrm{MnO}_{2}(\mathrm{~d})$ 纳米线的HRTEM图

催化剂作用的判断. 在反应体系中缺少 $\mathrm{MnO}_{2}$ 纳米线 或者反应体系溶液缺乏溶解氧的条件下, 生苂反应则 被大大地抑制, 甚至不发生. 这一结果表明, 溶解氧在 生苂反应中起到氧化剂的角色(图S7). 此外, 在相同的 实验条件下, 如果反应体系溶液中含有OPD、 $\mathrm{MnO}_{2}$ 纳 米线和溶解氧, 无论在避光还是非避光的条件下, 生 苂反应也均会发生(图S8). 由此可认为, 这种生苂反应 并不是由光催化作用所引起的. 此外, 通过电化学循 环伏安法测得 $\mathrm{MnO}_{2}$ 纳米线和 $\mathrm{OPD}$ 的氧化还原电位分 别为 -0.18 和 $0.25 \mathrm{~V}$. 因此, 进一步可由 $\Delta G=-n \Delta \varphi F$ 计算 出 Gibbs自由能 $\Delta G=41.195 \mathrm{~kJ} \mathrm{~mol}^{-1}$. 显然, $\Delta G>0$, 表明 $\mathrm{MnO}_{2}$ 纳米线不能作为氧化剂直接氧化 OPD (图S9和 $\mathrm{S} 10)$. 以上的实验结果均表明, $\mathrm{MnO}_{2}$ 纳米线在生荧反 应中起到催化剂作用.

在以上的研究基础上, 进一步以 $\beta-\mathrm{MnO}_{2}$ 纳米线为 催化剂, $\mathrm{OPD}$ 为底物分子, 分别考察了 $\mathrm{pH}$ 、温度、反应 时间、 $\mathrm{OPD}$ 和 $\beta-\mathrm{MnO}_{2}$ 纳米线浓度的影响, 从而优化催 化生苂反应的实验条件. 当反应体系 $\mathrm{pH}$ 从 3.8 增大到 8.0 时, $\beta-\mathrm{MnO}_{2}$ 纳米线的催化活性先增大后降低(图4(a)). 结合生物兼容性方面考虑, 采用 $\mathrm{pH}$ 7.4的 $0.2 \mathrm{M} \mathrm{PB}$ 缓冲 溶液进行后续实验. 反应温度效应实验在 $30 \sim 70^{\circ} \mathrm{C}$ 范 围内进行. 实验结果表明, 催化生苂反应的活性随温度 的上升而增大(图4(b)). OPD浓度效应实验揭示, 随着 OPD浓度的增加, 反应体系在最大发射波长 $564 \mathrm{~nm}$ 处 的荧光强度也增大, 并在OPD浓度达到 $0.075 \mathrm{mM}$ 时, 苂 光强度值出现一个平台区 (图4(c)). 催化剂 $\beta-\mathrm{MnO}_{2}$ 纳米 线的浓度效应也表明, $\beta-\mathrm{MnO}_{2}$ 纳米线浓度从 $0 \mu \mathrm{g} / \mathrm{mL}$ 增加到 $5.5 \mu \mathrm{g} / \mathrm{mL}$ 时, 体系的反应速率不仅会加快, 而 


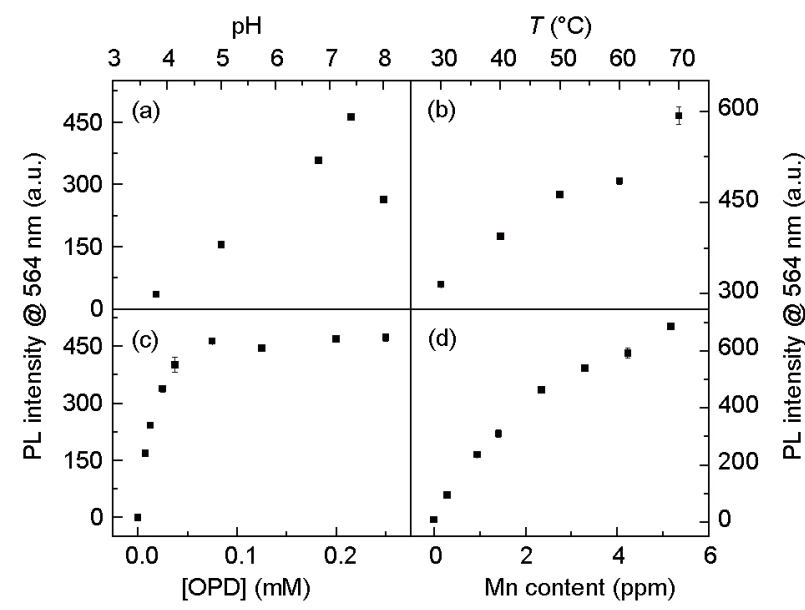

图 $4 \mathrm{pH}(\mathrm{a})$ 、温度 (b)、OPD浓度 $(\mathrm{c}) 、 \beta-\mathrm{MnO}_{2}$ 纳米线浓度 (d)对催化生荧反应体系的影响. 实验条件: $[\mathrm{OPD}]=0.075 \mathrm{mM}$; 温度 $50^{\circ} \mathrm{C} ; \mathrm{pH} 7.4$ 的 $0.2 \mathrm{M} \mathrm{PB}$ 缓冲溶液; $\left[\beta-\mathrm{MnO}_{2}\right]=2.5 \mu \mathrm{g} / \mathrm{mL}$ ( $\mathrm{Mn}$ 含量为2.35 ppm)

且在 $564 \mathrm{~nm}$ 处的苂光强度也会增大(图4(d)). 此外, 反应 时间效应的实验结果表明, 从 $0 \mathrm{~min}$ 到 $30 \mathrm{~min}$ 时, $564 \mathrm{~nm}$ 处的荧光强度增加较快, 但在 $30 \mathrm{~min}$ 后, 荧光强度变化 则比较缓慢(图S11). 因此, 综合考虑, 后续实验的温 度、OPD浓度、 $\beta-\mathrm{MnO}_{2}$ 纳米线浓度以及反应时间分 别选用 $50^{\circ} \mathrm{C} 、 0.075 \mathrm{mM} 、 2.5 \mu \mathrm{g} \mathrm{mL}^{-1}$ 和 $30 \mathrm{~min}$.

为了进一步探究 $\beta-\mathrm{MnO}_{2}$ 纳米线对 $\mathrm{OPD}$ 的催化生 荧反应, 利用电喷雾质谱(ESI-MS)研究了催化反应后 的产物. 实验结果揭示, OPD主要氧化产物的 $m / z$ 为 211.0979 (图5). 由此, 可推测该反应产物为2,3-二氨基 吩嗪. 核磁共振 $\left({ }^{1} \mathrm{H}\right.$ NMR $)$ 实验结果则进一步证实了这 种OPD氧化产物的分子结构(图6).

用初始速率法可求出催化生菼反应的表观稳态 动力学参数. 通过UV-Vis 吸收光谱, 由OPD的氧化产 物(2,3-二氨基吩嗪, 缩写为OPDox, 商业化可得)的标 准浓度曲线, 可计算出催化生菼反应体系中生成OPD 氧化产物的浓度. 进一步由 $\ln \left(C_{t} / C_{0}\right)-t$ 关系曲线, 可获 得反应速率常数. 由此, 可作出Michaelis-Menten方程 曲线及其双倒数曲线(图7), 从而计算得到催化反应参 数(表 $\mathrm{S} 1)$. 该催化生荧反应体系中的表观米氏常数 $K_{\mathrm{m}}$ 小于辣根过氧化物酶(HRP) ${ }^{[4]}$ 的, 表明 $\beta-\mathrm{MnO}_{2}$ 纳米线 比HRP对OPD的吸附能力更强.

根据Arrhenius方程可测得不同温度条件下 $\beta-\mathrm{MnO}_{2}$ 纳米线催化OPD生荧反应的活化能:

$$
\ln k=\ln A-E_{\mathrm{a}} / R T
$$

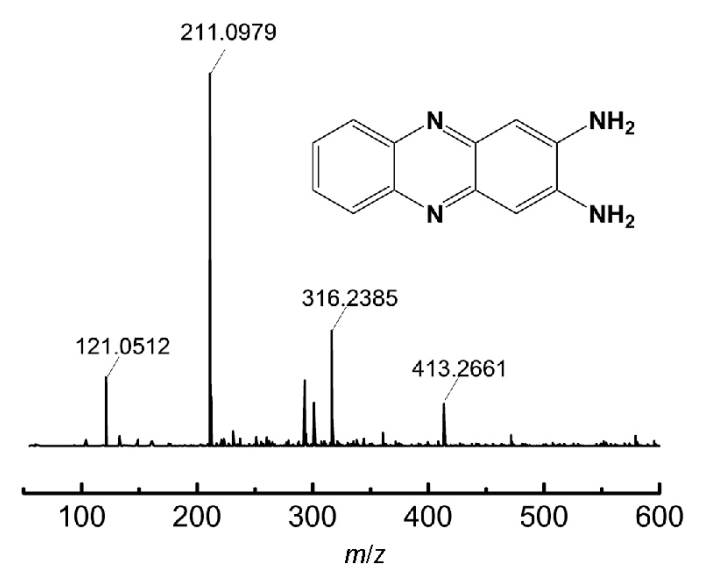

图 $5 \beta-\mathrm{MnO}_{2}-\mathrm{OPD}$ 催化反应体系的ESI-MS实验结果图. 主 要产物可归属于 $2,3-$ 二氨基吩嗪 $\left([\mathrm{M}+\mathrm{H}]^{+}\right)$

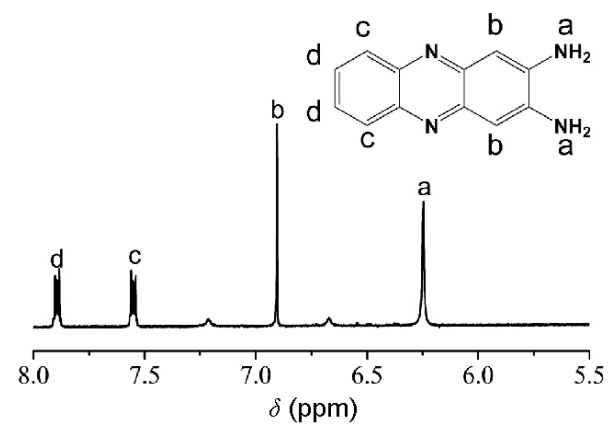

图 $6 \mathrm{DMSO}-d_{6}$ 作溶剂的 $\beta-\mathrm{MnO}_{2}$ 纳米线催化 $\mathrm{OPD}$ 氧化产物 的 $\mathrm{H}^{1} \mathrm{NMR}(500 \mathrm{MHz})$ 图

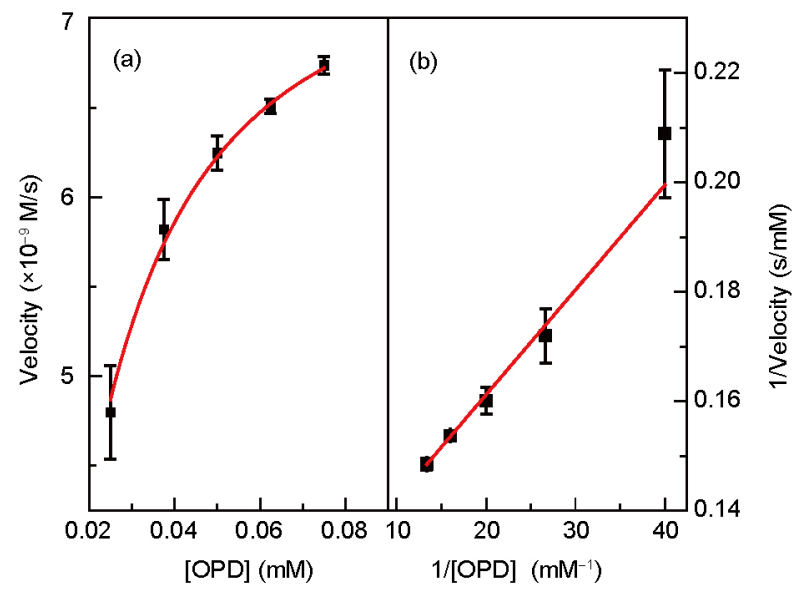

图 $7 \beta-\mathrm{MnO}_{2}$ 纳米线催化 $\mathrm{OPD}$ 氧化反应Michaelis-Menten模 型的稳态动力学分析 (网络版彩图) 
其中 $A$ 为指前因子, $k$ 为表观速率常数, $T$ 为系统的绝对 温度, $E_{\mathrm{a}}$ 为活化能, $R$ 为理想气体常数.

在优化的实验条件下, 以温度为变量 $(40 、 50 、 60$ 和 $\left.70^{\circ} \mathrm{C}\right)$, 测定各温度下的催化生荧反应速率常数 $k$, 并 用 $\ln k$ 对 $1000 / T$ 作图, 结果参见图S12. 由图S12可知, 随 着温度升高, 反应速率常数增大. 经拟合后获得直线 的斜率即为 $E_{\mathrm{a}} / R$. 由此, 可计算出该催化生苂反应体系 的活化能 $E_{\mathrm{a}}=28.31 \mathrm{~kJ} \mathrm{~mol}^{-1}$, 表明 $\beta-\mathrm{MnO}_{2}$ 纳米线具有高 催化活性.

研究 $\beta-\mathrm{MnO}_{2}$ 纳米线催化 $\mathrm{OPD}$ 的生苂反应, 为进一 步探究其在分析化学方面的扩展应用打下基础. 甲醛 常被非法地添加进食品中作为保鲜剂, 而人体过量地 摄入甲醛可能会引起相关的癌症疾病 ${ }^{[3]}$. 因此, 发展一 种简便、灵敏和高选择性的分析方法用于检测食品中 的甲醛非常重要. 目前, 已经构建有电化学、UV-Vis 吸收光谱、苂光光谱、毛细管电泳等方法用于检测 食品中的甲醛 ${ }^{[34-37]}$. 考虑到甲醛的-CHO基团和 OPD 的 $-\mathrm{NH}_{2}$ 基团容易发生缩合反应生成Schiff碱. 因此, 当 将一定数量的甲醛加入到 $\beta-\mathrm{MnO}_{2}$ 纳米线催化生荧反 应体系中时, 该催化生苂反应将会受到抑制, 从而达 到传感甲醛之目的(图8). 实验结果证实了这一推断, 发现在所考察的甲醛浓度范围内, 甲醛浓度与564 nm 波长处的苂光强度呈良好的线性关系, 并且检测限为 $2.95 \mu \mathrm{M}(3 \sigma / k, n=11)$.

在相同的实验条件下, 一系列常见的金属离子、 阴离子、有机小分子等干扰物质的浓度是甲醛浓度 的10倍时, 均未对该催化生苂反应产生明显的抑制作 用(图9). 由此表明, 该催化苂光传感体系具有较高的 选择性. ESI-MS实验进一步研究发现, OPD与甲醛经 缩合反应生成可归属为1-甲基苯并咪唑 $(m / z$ 133.077) 和苯并咪唑 $(m / z$ 119.062)的产物(图S13).

为了探究所构建的传感体系在实际应用中的可 行性, 拟将其应用于海产品样品中甲醛的回收率的测 定研究. 在实验中, 选择市场上常见的散装干鱿鱼作 为实际样品, 进行甲醛回收率的测定实验. 实验结果 见表1. 90.1\% 110.4\%的回收率表明, 该催化生荧传感 体系具有良好的实际应用意义. 甲醛的- $\mathrm{CHO}$ 基团与 催化底物分子 $\mathrm{OPD}$ 的 $-\mathrm{NH}_{2}$ 基团经缩合反应生成Schiff 碱, 保障了该催化生苂传感体系的高选择性. 因此, 与 传统的荧光光谱、UV-Vis吸收光谱法比较, 本文所构 筑的甲醛催化生苂传感体系具有简单、选择性高、

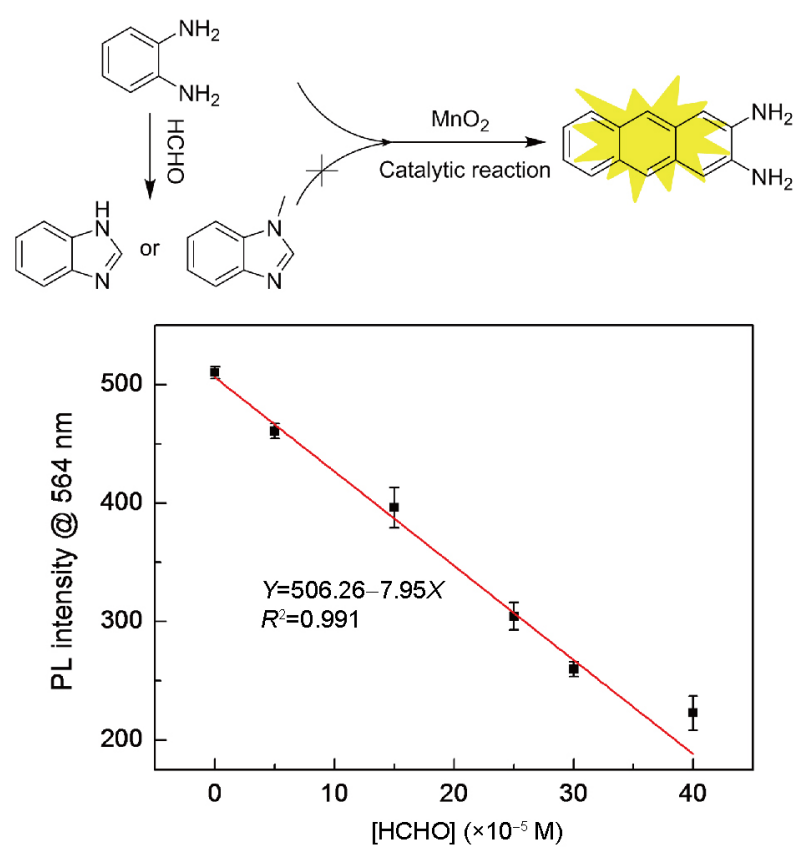

图 8 基于 $\beta-\mathrm{MnO}_{2}$ 纳米线催化 $\mathrm{OPD}$ 生苂反应的甲醛传感机 理以及苂光响应工作曲线(网络版彩图)

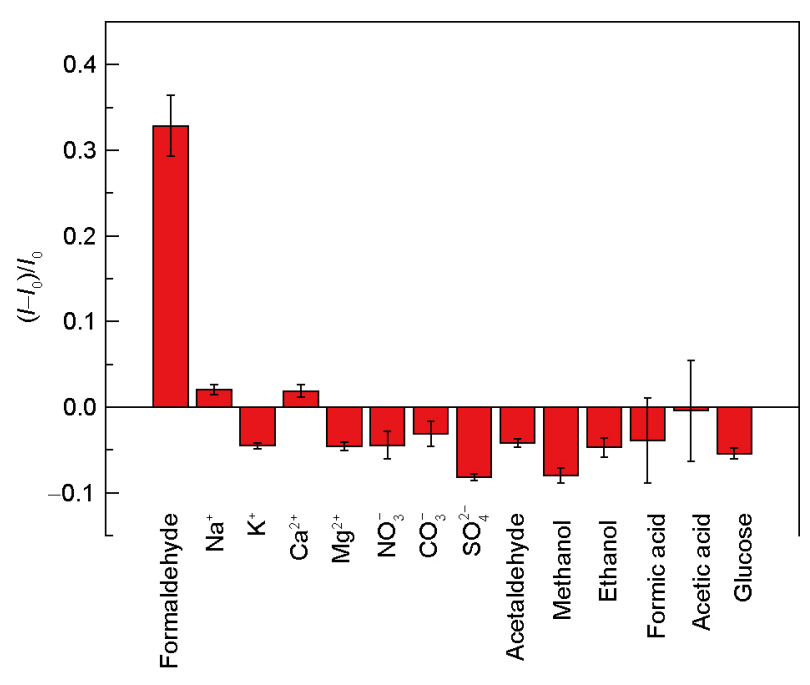

图 $9 \beta-\mathrm{MnO}_{2}$ 纳米线催化 $\mathrm{OPD}$ 生苂反应的选择性

表 1 干鱿鱼样品中甲醛的回收率实验结果 ${ }^{a)}$

\begin{tabular}{cccc}
\hline No. & Spiked $\left(10^{-5} \mathrm{M}\right)$ & Measured $\left(10^{-5} \mathrm{M}\right)$ & Recovery $(\%)$ \\
\hline 1 & 0 & $0.2 \pm 0.19$ & \\
2 & 4 & $3.81 \pm 0.22$ & $90.1 \pm 0.08$ \\
3 & 6 & $6.83 \pm 0.13$ & $110.4 \pm 0.05$ \\
\hline
\end{tabular}

a)所有实验平行 3 组. 
无需合成复杂的受体分子等优点 ${ }^{[38-41]}$.

\section{4 结论}

通过水热合成法, 制备了 4 种不同晶型 $(\alpha 、 \beta 、 \gamma 、$ $\delta)$ 的 $\mathrm{MnO}_{2}$ 纳米线, 以常见的酶底物 $\mathrm{OPD}$ 为底物分子, 分别研究了其催化生荧反应. 经对比, $\beta-\mathrm{MnO}_{2}$ 纳米线 的催化生荧反应活性最优, 这可能是与 $\beta-\mathrm{MnO}_{2}$ 纳米线 由(110)晶面占主导, 而 $\alpha-、 \gamma-、 \delta-\mathrm{MnO}_{2}$ 纳米线的主导 晶面不明显有关. 在优化的实验条件下, 利用稳态动 力学研究获得 $\beta-\mathrm{MnO}_{2}$ 纳米线对 $\mathrm{OPD}$ 催化生苂反应的
动力学参数, 并由质谱和核磁共振实验结果可推断出 催化生荧反应的主要产物结构. 在此研究基础上, 借 助甲醛的- $\mathrm{CHO}$ 基团和底物分子 $\mathrm{OPD}$ 的 $-\mathrm{NH}_{2}$ 基团经缩 合反应生成Schiff碱, 从而抑制催化生荧反应的性质, 构建了一种新型的甲醛催化苂光传感方法. 与已报道 的甲醛检测方法相比, 本文所构建的催化生荧传感体 系具有简便、选择性更高、无需合成复杂的受体分 子等优点. 此外, 本文的研究结果不仅有利于发展新 型的催化传感体系, 而且对具有不同晶型纳米材料的 催化反应研究提供一定启示.

\section{支持信息}

本文的支持信息见网络版chem.scichina.com. 支持信息为作者提供的原始数据,作者对其学术质量和内容负责.

\section{参考文献}

朱圣庚, 徐长法. 生物化学. 第4版. 北京: 高等教育出版社, 2017

Wei H, Wang E. Chem Soc Rev, 2013, 42: 6060-6093

Wang X, Hu Y, Wei H. Inorg Chem Front, 2016, 3: 41-60

Gao L, Zhuang J, Nie L, Zhang J, Zhang Y, Gu N, Wang T, Feng J, Yang D, Perrett S, Yan X. Nat Nanotech, 2007, 2: 577-583

Chen X, Tian X, Su B, Huang Z, Chen X, Oyama M. Dalton Trans, 2014, 43: 7449-7454

Jiang H, Chen Z, Cao H, Huang Y. Analyst, 2012, 137: 5560-5564

Hu L, Yuan Y, Zhang L, Zhao J, Majeed S, Xu G. Anal Chim Acta, 2013, 762: 83-86

Chen W, Chen J, Liu AL, Wang LM, Li GW, Lin XH. ChemCatChem, 2011, 3: 1151-1154

Asati A, Santra S, Kaittanis C, Nath S, Perez JM. Angew Chem Int Ed, 2009, 48: 2308-2312

Chaudhari KN, Chaudhari NK, Yu JS. Catal Sci Technol, 2012, 2: 119-124

Dutta AK, Maji SK, Srivastava DN, Mondal A, Biswas P, Paul P, Adhikary B. ACS Appl Mater Interfaces, 2012, 4: 1919-1927

He W, Jia H, Li X, Lei Y, Li J, Zhao H, Mi L, Zhang L, Zheng Z. Nanoscale, 2012, 4: 3501-3506

Song Y, Wang X, Zhao C, Qu K, Ren J, Qu X. Chem Eur J, 2010, 16: 3617-3621

Shi W, Wang Q, Long Y, Cheng Z, Chen S, Zheng H, Huang Y. Chem Commun, 2011, 47: 6695-6697

Song Y, Qu K, Zhao C, Ren J, Qu X. Adv Mater, 2010, 22: 2206-2210

Liu S, Lu F, Xing R, Zhu JJ. Chem Eur J, 2011, 17: 620-625

Shen JS, Wu JJ, Sun XY, Wu ZL, Gao P, Liu B. J Mater Chem C, 2017, 5: 3757-3764

李莉, 魏子栋, 李兰兰, 孙才新. 化学学报, 2006, 64: 287-294

Jana S, Basu S, Pande S, Ghosh SK, Pal T. J Phys Chem C, 2007, 111: 16272-16277

Xie Y, Yu Y, Gong X, Guo Y, Guo Y, Wang Y, Lu G. CrystEngComm, 2015, 17: 3005-3014

Débart A, Paterson AJ, Bao JL, Bruce PG. Angew Chem, 2008, 120: 4597-4600

Hu Y, Zhang T, Cheng F, Zhao Q, Han X, Chen J. Angew Chem Int Ed, 2015, 54: 4338-4343

Zhai W, Wang C, Yu P, Wang Y, Mao L. Anal Chem, 2014, 86: 12206-12213

Wang X, Li Y. Chem-A Eur J, 2003, 9: 300-306

Zhang J, Li Y, Wang L, Zhang C, He H. Catal Sci Technol, 2015, 5: 2305-2313

Liu X, Wang Q, Zhao H, Zhang L, Su Y, Lv Y. Analyst, 2012, 137: 4552-4558

Banerjee D, Nesbitt HW. GeoChim CosmoChim Acta, 1999, 63: 3025-3038

Nesbitt HW, Banerjee D. Am Miner, 1998, 83: 305-315

Subramanian V, Zhu H, Vajtai R, Ajayan PM, Wei B. J Phys Chem B, 2005, 109: 20207-20214

Wan Y, Qi P, Zhang D, Wu J, Wang Y. Biosens Bioelectron, 2012, 33: 69-74 


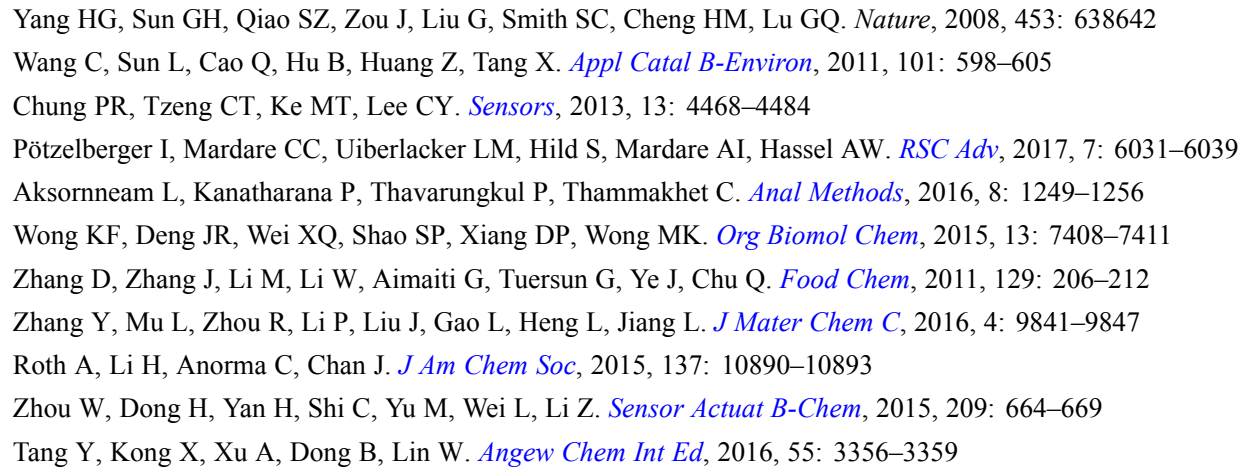

\title{
Fluorescence sensing of formaldehyde based on the catalytic reaction of $\mathrm{MnO}_{2}$ nanowires modulated by different crystal structures
}

\author{
Zhenlong Wu, Jiajia Wu, Xiangying Sun, Bin Liu, Jiangshan Shen* \\ College of Materials Science and Engineering, Huaqiao University, Xiamen 361021, China \\ *Corresponding author (email: jsshen@hqu.edu.cn)
}

\begin{abstract}
MnO}_{2}$ nanowires with different crystal structures were synthesized by a hydrothermal method. Employing $o$-phenylenediamine (OPD) as the substrate, the fluorogenic reactions catalyzed by these $\mathrm{MnO}_{2}$ nanowires were detailedly studied. Transmission electron microscopy (TEM), scanning electron microscopy (SEM), X-ray diffraction (XRD), X-ray photoelectron spectroscopy (XPS), nuclear magnetic resonance (NMR), mass spectrometry and electrochemical methods were employed to probe the catalytic fluorogenic reaction. Experimental results revealed that, the activity of the catalytic fluorogenic reaction of $\beta-\mathrm{MnO}_{2}$ nanowires towards OPD was best, compared with that of other $\mathrm{MnO}_{2}$ nanowires with different crystal structures. On the basis of these findings, taken together the condensation reaction forming the Schiff base between the $-\mathrm{CHO}$ group of formaldehyde and $-\mathrm{NH}_{2}$ group of $\mathrm{OPD}$, a novel, highly selective and sensitive catalytic fluorogenic sensing system was successfully established and employed for detecting formaldehyde in foods.
\end{abstract}

Keywords: $\mathrm{MnO}_{2}$ nanowires, the catalytic fluorogenic reaction, fluorescence sensing, detecting formaldehyde doi: $10.1360 / \mathrm{N} 032017-00072$ 